\title{
Malignant, but not benign, intraductal papillary mucinous neoplasm preferentially associates with prior extrapancreatic malignancies
}

\author{
TAKAHARU KATO ${ }^{1,2}$, SERGIO ALONSO ${ }^{2}$, HIROSHI NODA ${ }^{1}$, YASUYUKI MIYAKURA ${ }^{1}$, \\ SHINGO TSUJINAKA ${ }^{1}$, MASAAKI SAITO ${ }^{1}$, YUTA MUTO $^{1}{ }^{1}$, TARO FUKUI ${ }^{1}$, \\ KOSUKE ICHIDA $^{1}$, YUJI TAKAYAMA ${ }^{1}$, FUMIAKI WATANABE ${ }^{1}$, NAO KAKIZAWA ${ }^{1}$, \\ MANUEL PERUCHO $^{2-4}$ and TOSHIKI RIKIYAMA ${ }^{1}$
}

\footnotetext{
${ }^{1}$ Department of Surgery, Saitama Medical Center, Jichi Medical University, Omiya-ku, Saitama 330-8503, Japan;

${ }^{2}$ Institute of Predictive and Personalized Medicine of Cancer (IMPPC), Institut d'Investigació en Ciéncies de la Salut Germans Trias I Pujol, (IGTP), Campus Can Ruti, 08916 Badalona, Barcelona, Spain;

${ }^{3}$ Sanford-Burnham-Prebys Medical Discovery Institute, La Jolla, CA 92037, USA;

${ }^{4}$ Institució Catalana de Recerca I Estudis Avançats (ICREA), 08010 Barcelona, Spain
}

Received December 18, 2015; Accepted February 9, 2016

DOI: 10.3892/or.2016.4755

\begin{abstract}
Intraductal papillary mucinous neoplasm (IPMN) has been associated with a high incidence of extrapancreatic malignancies (EPMs). However, it is controversial whether IPMN is prognostic for EPM. We aimed to help clarify the issue studying this association in patients with histologically proven IPMN. We reviewed 51 surgically resected IPMNs in Saitama Medical Center, Jichi Medical University between January 1991 and June 2012. Mean follow-up was $63.7 \pm 47.8$ months. The observed EPM incidence was compared with the expected incidence of cancer in Japan. Of the 51 IPMNs, 14 were malignant and the rest benign. Seventeen EPMs developed in 15 patients (29.4\%), nine of which occurred prior to IPMN diagnosis. For all IPMNs, the standardized incidence ratio (SIR) was significantly increased for the six types of reported EPMs ( $\mathrm{SIR}=2.18, \mathrm{CI}=1.31-3.42, \mathrm{P}=0.004)$. Benign IPMNs showed no association with EPMs (SIR=0.92, CI=0.43-1,76, $\mathrm{P}=0.87$ ). In contrast, malignant IPMNs showed a higher association ( $\mathrm{SIR}=3.83, \mathrm{CI}=1.87-7.03, \mathrm{P}=0.0009)$. However,
\end{abstract}

Correspondence to: Dr Takaharu Kato, Department of Surgery, Saitama Medical Center, Jichi Medical University, 1-847 Amanuma-cho, Omiya-ku, Saitama 330-8503, Japan E-mail: tkato@jichi.ac.jp

Abbreviations: IPMN, intraductal papillary mucinous neoplasm; EPM, extra-pancreatic malignancy; SIR, standard incidence ratio; PDAC, pancreatic ductal adenocarcinoma

Key words: intraductal papillary mucinous neoplasms, intraductal papillary mucinous cancer, extrapancreatic malignancy, pancreas, gastrointestinal cancer the association was mostly due to the prior EPMs, as removal of metachronous EPMs had no significant effect ( $\mathrm{SIR}=3.63$, $\mathrm{CI}=1.59-7.17, \mathrm{P}=0.005)$. Thus, only malignant IPMNs drive the significant association with prior EPMs, showing a near 4-fold increased incidence compared to the general Japanese population. Histological characterization of IPMNs may offer clinical value for EPM patient management. We hypothesize that these observations may be explained if some patients with EPMs present a higher risk to develop IPMNs (and vice versa), possibly resulting from an uncharacterized multiple cancer predisposition condition.

\section{Introduction}

Recently, intraductal papillary mucinous neoplasm (IPMN) has gathered increased attention because of its favorable prognosis despite its association with pancreatic cancer (1-3). IPMN has been associated with extra pancreatic malignancies (EPMs). Since the first report (1), several studies described that between 17 and $48 \%$ of IPMN patients are diagnosed with EPM (4-9). In all these studies, the incidence of IPMN-related EPMs exceeded the expected rate of such malignancies in the general population. Consequently, it has been generally accepted that IPMN patients have an increased risk for EPMs. Two recent investigations, however, did not find any significant association between IPMNs and the subsequent development of EPMs, questioning whether IPMNs are actually a risk factor for EPM development $(10,11)$.

Understanding the IPMN characteristics and the associated EPMs is important in order to administer appropriate treatment and to determine suitable follow-up plans for these patients. Our study was aimed to help clarify the clinical and pathological features of EPMs and their association with IPMNs in Japanese patients. We reviewed medical records of histologically proven IPMN from patients who underwent 
pancreatic surgery and compared the observed with the expected frequency of EPMs in the Japanese population.

\section{Materials and methods}

Study population. Between January 1991 and June 2012, 51 patients underwent surgical resection for IPMN at Saitama Medical Center, Jichi Medical University. Medical records were reviewed retrospectively for patient characteristics, perioperative clinical data, operative management, pathology examination results (12), and postoperative course. History of pre-existent or concurrent EPMs was investigated thoroughly. Follow-up information, including postoperative development of EPMs, was obtained by reviewing outpatient medical records. A positive family history was defined as a history of malignancy among one or more first-degree relatives. Mean follow-up was $63.7 \pm 47.8$ months and mean patient age was $68.0 \pm 7.2$ years.

Definition of EPM. In accordance with the International Agency for Research on Cancer (13), EPMs were defined as malignancies in an organ other than pancreas, not being an infiltration from another neoplasm, a relapse or a metastasis, regardless the time of occurrence. EPMs developing before or within a time span of \pm 6 months from the time of IPMN resection were classified as pre-existent or concomitant, respectively. EPMs diagnosed 6 months after IPMN surgery were classified as metachronous. If a patient developed two or more EPM malignancies they were counted independently.

Calculation method of standard incidence ratio (SIR). SIR was calculated as the ratio of the observed to the expected number of patients developing EPMs (9). The expected number was determined using age-stratified and sex-specific data on the incidence of cancer in Japan, provided by the Center for Cancer Control and Information Services, National Cancer Center Japan (14). Age at the end of the follow-up period, or age at the time of diagnosis of EPM, was employed to determine the expected incidence for every patient.

Statistical analysis. Categorical variables were compared using Fisher's exact test. Continuous variables were compared using t-test or Wilcoxon-Mann-Whitney test. Deviation from normality was determined by Shapiro's test. Kaplan-Meier model with log-rank test was used to assess differences in survival. Differences were considered statistically significant at $\mathrm{P}<0.05$. Statistical analyses were performed using the $\mathrm{R}$ environment for statistical computing and the OpenEpi statistical calculator $(15,16)$.

Ethics statement. In this retrospective study, the clinical information on patients from the Saitama Medical Center, Jichi Medical University was anonymized. The study was approved by the Research Ethics Committee, complying with the ethical guidelines of the Declaration of Helsinki (17).

\section{Results}

Fourteen of 51 patients had invasive carcinomas of malignant IPMN (malignant IPMNs) and 37 had mild to high grade
Table I. EPMs in patients with IPMN.

\begin{tabular}{lcccc}
\hline Organ & $\begin{array}{c}\text { Pre- } \\
\text { existent }\end{array}$ & Synchronous & Metachronous & Total \\
\hline Colorectal cancer & 4 & 2 & 0 & 6 \\
Stomach cancer & 2 & 1 & 1 & 4 \\
Lung cancer & 0 & 0 & 2 & 2 \\
Breast cancer & 2 & 0 & 0 & 2 \\
Hepatic cancer & 0 & 0 & 2 & 2 \\
Renal cancer & 1 & 0 & 0 & 1 \\
Total & 9 & 3 & 5 & 17 \\
\hline
\end{tabular}

dysplasias derived from IPMN (benign IPMN). Survival rates of IPMN patients at 5 years after surgery were $74.3 \%$ overall, $86.5 \%$ for benign, and $49.0 \%$ for malignant IPMN ( $\mathrm{P}=0.005$; log-rank test comparing benign vs. malignant IPMN). During postoperative follow-up of 51 IPMN patients that underwent surgical resection, 7 patients died of malignant IPMN, 5 patients died of non-malignant diseases, and other 2 died of uncertain cause. Seventeen EPMs presented in 15 of the 51 IPMN patients $(29.4 \%$, Table I). The most frequent EPM was colorectal cancer ( 6 cancers in 6 patients, $40 \%$ ), followed by stomach cancer (4 cancers in 4 patients, 26.7\%). Among the 17 EPMs, 9 were pre-existent, diagnosed 15 to 348 months (mean, 140.7 months) before the surgery for IPMN, 3 EPMs were diagnosed concurrently, and 5 were diagnosed 12 to 139 months (mean, 51.7) after surgery (Table I). One patient had developed both stomach and colon cancer synchronously 48 months before the surgery for IPMN. Another patient had previously developed bilateral breast cancer, 20 years before surgery for IPMN. One patient did not develop EPM but an invasive ductal pancreatic cancer (PDAC) 26 months after IPMN resection.

Comparison of clinicopathological features of IPMN patients with and without EPM revealed no statistically significant differences in age, gender, follow-up, family history of malignancy, history of cigarette smoking or alcohol consumption, type of IPMNs (branch, mix or main duct type), or survival (Table II).

SIR were calculated for 50 IPMN patients, after excluding the patient that developed PDAC during follow-up. The incidence of gastrointestinal EPMs (esophageal, gastric, hepatic, gallbladder, bile duct and colorectal malignancies) was also significantly higher $(\mathrm{SIR}=2.11, \mathrm{CI}=1.14-3.58, \mathrm{P}=0.02)$. Considering together the six types of EPM found in these patients, i.e., colorectal, gastric, lung, breast, hepatic and renal cancer, the incidence was also significantly higher than expected ( $\mathrm{SIR}=2.18, \mathrm{CI}=1.31-3.42, \mathrm{P}=0.004)$. The $\mathrm{SIR}$ for EPMs occurring in all organs was elevated but did not reach significance $(\mathrm{SIR}=1.44, \mathrm{CI}=0.87-2.21, \mathrm{P}=0.15)$ (Table III).

No statistically significant increase in EPM incidence was found for patients with benign IPMN (Table III). In contrast, the EPM incidence in malignant IPMN patients was elevated in colon, stomach, breast, lung, liver and kidney when considered individually (SIR between 2.3 and 12.3; data not shown), albeit it did not reach statistical significance for any of them. The incidence of gastrointestinal EPMs was significantly 
Table II. Clinicopathological features of IPMN patients with and without EPMs.

\begin{tabular}{|c|c|c|c|}
\hline & With EPM $(\mathrm{n}=15 ; 29.4 \%)$ & Without EPM (n=36; 70.6\%) & P-value \\
\hline \multicolumn{4}{|l|}{ Age (years), mean \pm SD } \\
\hline At operation & $69.3 \pm 6.8$ & $66.8 \pm 7.3$ & $0.25^{\mathrm{b}}$ \\
\hline At end of follow-up & $73.9 \pm 7.2$ & $72.7 \pm 7.0$ & $0.59^{\mathrm{b}}$ \\
\hline Gender (male/female), no. & $8 / 7$ & $21 / 15$ & 0.77 \\
\hline Follow-up (months) mean \pm SD & $54.8 \pm 38.9$ & $67.4 \pm 51.2$ & $0.47^{\mathrm{c}}$ \\
\hline Family history of cancer, no. (\%) & $7(46.7)$ & $14(38.9)$ & 0.76 \\
\hline Smoking, no. (\%) & $9(60.0)$ & $21(58.3)$ & 1 \\
\hline Alcohol, no. $(\%)$ & $9(60.0)$ & $19(52.8)$ & 0.76 \\
\hline Type of IPMN, no. (\%) & & & 0.95 \\
\hline Main duct & $6(40.0)$ & $14(38.9)$ & \\
\hline Branch duct & $5(33.3)$ & $14(38.9)$ & \\
\hline Mixed & $4(26.7)$ & 7 (19.4) & \\
\hline Not classified & $0(0.00)$ & $1(2.8)$ & \\
\hline Location of IPMN lesion, no. $(\%)^{\mathrm{e}}$ & & & 0.16 \\
\hline Proximal & $10(66.7)$ & $29(80.6)$ & \\
\hline Distal & $4(26.7)$ & $4(11.1)$ & \\
\hline Total pancreas & $0(0.0)$ & $3(8.3)$ & \\
\hline Unknown & $1(6.7)$ & $0(0.0)$ & \\
\hline IPMN histology, no. (\%) & & & 0.083 \\
\hline Mild to high grade dysplasia & $8(53.3)$ & $29(81.6)$ & \\
\hline Invasive carcinoma & $7(46.7)$ & $7(19.4)$ & \\
\hline Survival ratio (years) $(\%)$ & & & $0.93^{\mathrm{d}}$ \\
\hline 5 & $(77.0)$ & $(73.5)$ & \\
\hline 10 & $(66.0)$ & $(62.9)$ & \\
\hline
\end{tabular}

Values in parentheses are percentages. ${ }^{a}$ Fisher's exact test, except ${ }^{b}$ Student's t-test, ${ }^{\mathrm{c} M a n n-W h i t n e y ~ U}$ test and ${ }^{\mathrm{d}} \log$-rank test. ${ }^{\mathrm{e}} \mathrm{Proximal}$, head or head and body lesions; distal, body and/or tail lesions.

elevated $(\mathrm{SIR}=3.0, \mathrm{CI}=1.10-6.66, \mathrm{P}=0.035)$ as well as for all six reported EPMs $(\mathrm{SIR}=3.83, \mathrm{CI}=1.87-7.03, \mathrm{P}=0.0009)$. The incidence for EPMs considering all organs was also significantly elevated compared with that of the general Japanese population ( $\mathrm{SIR}=2.53$, CI 1.23-4.64, $\mathrm{P}=0.015$ ) (Table III). The associations remained significant after excluding IPMN patients with metachronous EPMs (Table III).

\section{Discussion}

The main novel finding of our study is that EPMs were more frequent in malignant $(7 / 14,50.0 \%)$ than in benign $(8 / 36,21.6 \%)$ IPMN patients. Benign IPMN patients did not exhibit statistically significant higher incidence of EPM for any specific target organ, or when all the target organs were considered together. However, when considering malignant IPMN patients, the overall incidence of EPM in the reported target organs was significantly higher than in the reference Japanese population. This was also the case for EPMs affecting the gastrointestinal tract, and when considering EPM in any target organ (Table III). Precise histological characterization of IPMNs, for instance by endoscopy brushing biopsies, may offer clinical benefits to better assess the potential EPM incidence in IPMN patients, and thus improving patient management.

We are aware of some limitations of our study that will require further verification. The number of patients recruited was limited because of the low incidence of this type of lesions and because we purposely selected patients that underwent surgery to have a reliable histological classification of the IPMN. Also, in this retrospective study information of prior cancer history may have been missed or not clearly documented. But if that were the case, we would be underestimating, rather than overestimating, the incidence of EPMs in IPMN patients.

Two recent reports suggested IPMN is not a risk factor for developing EPMs $(10,11)$. Both were prospective studies in which patients with pre-existing or concomitant EPMs were excluded. In our view, their data convincingly indicate that the non-invasive detection of IPMN in cancer-naive individuals is not predictive of future development of EPMs. Nevertheless, our data and other previous studies show a clear association between IPMN and EPM (1,4-9). To exclude patients with concomitant (synchronous) EPMs may have important 
Table III. Standardized incidence ratio of EPM in IPMN patients.

\begin{tabular}{|c|c|c|c|c|c|}
\hline & Observed & Expected & SIR & P-value & $95 \% \mathrm{CI}$ \\
\hline \multicolumn{6}{|l|}{ All IPMN (n=50) } \\
\hline Total EPMs reported $^{\mathrm{a}}$ & 17 & 7.80 & 2.18 & 0.004 & $1.31-3.42$ \\
\hline Gastrointestinal $^{\mathrm{b}}$ & 12 & 5.70 & 2.11 & 0.02 & $1.14-3.58$ \\
\hline All organs ${ }^{c}$ & 17 & 11.81 & 1.44 & 0.15 & $0.87-2.21$ \\
\hline \multicolumn{6}{|l|}{ Benign IPMN (n=36) } \\
\hline Total EPMs reported $^{\mathrm{a}}$ & 8 & 5.46 & 1.47 & 0.29 & $0.68-2.78$ \\
\hline Gastrointestinal $^{\mathrm{b}}$ & 7 & 4.04 & 1.74 & 0.17 & $0.76-3.43$ \\
\hline All organs ${ }^{c}$ & 8 & 8.25 & 0.97 & 0.98 & $0.45-1.84$ \\
\hline \multicolumn{6}{|l|}{ Malignant IPMN (n=14) } \\
\hline Total EPMs reported ${ }^{\mathrm{a}}$ & 9 & 2.35 & 3.83 & 0.0009 & $1.87-7.03$ \\
\hline Gastrointestinal $^{\mathrm{b}}$ & 5 & 1.66 & 3.00 & 0.035 & $1.10-6.66$ \\
\hline All organs ${ }^{c}$ & 9 & 3.56 & 2.53 & 0.015 & $1.23-4.64$ \\
\hline \multicolumn{6}{|c|}{ Malignant IPMN excluding metachronous EPMs $(n=12)$} \\
\hline Total EPMs reported ${ }^{\mathrm{a}}$ & 7 & 1.93 & 3.63 & 0.005 & $1.59-7.17$ \\
\hline Gastrointestinal $^{\mathrm{b}}$ & 4 & 1.35 & 2.96 & 0.06 & $0.94-7.15$ \\
\hline All organs ${ }^{c}$ & 7 & 2.91 & 2.41 & 0.04 & $1.05-4.76$ \\
\hline
\end{tabular}

${ }^{\mathrm{a}}$ Total reported EPMs, colorectal, gastric, lung, breast, liver and renal EPMs. ${ }^{\mathrm{b}}$ Considering all gastrointestinal malignancies except pancreatic, esophageal, gastric, liver, gallbladder, bile duct and colorectal EPMs. ${ }^{\circ}$ Reported incidence for malignancies at all organs. SIR, standardized incidence ratio. Confidence intervals (CI) and P-values were calculated using the Mid-P method. In bold, P-values $<0.05$

repercussions in the estimation of the IPMN association with EPM. For instance, in the study if Kawakubo et al of the 642 IPMN patients with follow-up, 66 with concomitant EPMs $(10.3 \%)$ and 101 with pre-existent EPM (15.7\%) were excluded from the prospective analysis. If the 66 concomitantEPM patients were not excluded, assuming that their age and gender distribution were not significantly different from the 475 included in the prospective analysis, the SIR for EPM would have increased from the reported 0.94 (CI 0.67-1.29) to 2.23 (CI 1.83-2.69, P<0.001).

In contrast with our study, in the above two studies most patients did not undergo surgery for IPMN and thereby histological diagnosis was not determined. In general, IPMN patients receive surgery if they show some symptoms suggesting the presence of malignant IPMN according to standardized criteria (12). It is therefore probable that the majority of the non-operated patients included in these two studies had benign rather than malignant IPMN. Our results may explain the absence of association in these studies of the overall sample of IPMNs (the benign majority masking the contribution of the malignant minority) with EPMs.

Malignant IPMN patients frequently develop invasive ductal pancreatic cancer. Even when excluding these patients, the prognosis of malignant IPMN patients is worse than those with benign IPMN. Malignant IPMN patients may die before developing EPMs, thus reducing the number of metachronous EPMs observed in this group. Nevertheless, the association of malignant IPMNs with EPMs was mostly due to the pre-existent EPMs because there was no statistically significant increase in EPM incidence after excluding these patients regardless of the IPMN histological classification. Conversely, in malignant IPMN patients, the incidence of EPM in any target organ was still significantly higher than that in the reference Japanese population after metachronous EPMs were excluded (Table III).

We therefore, interpret these results as indicative that IPMNs, and especially malignant IPMNs, rather than predicting the future development of metachronous neoplasms, reflect a prior susceptibility or higher risk for the development of neoplastic disease. This is in line with the observation that second, independent cancers occur at higher rates in patients who have survived a primary cancer than in a cancer-naive population $(18,19)$. Previous reports suggested some common genetic or epigenetic risk factors in patients with IPMN and EPMs, such as field carcinogenesis, KRAS, TP53, and MUC2 mutations $(1,8,20-22)$, but the cause of frequent development of EPMs in patients with IPMN (or vice-versa) is unknown.

The increased prevalence of EPMs in malignant IPMN patients suggests the involvement of systemic/germline carcinogenic mechanisms or common environmental risk factors in these patients, as already indicated by the multistage character of the transformation of benign into malignant IPMN $(23,24)$. In other words, these patients may have been subjected to the effect of a cancer-risk factor, genetic/epigenetic alteration or environmental, that predisposed them to develop EPM as well as favored the malignant transformation of IPMN. Because the age of these patients is well above the age of known hereditary cancer syndromes, the clustering of these multiple neoplasms does not seem to be due to underlying genetic defects with high penetrance, typical of hereditary cancer syndromes, but rather to uncharacterized cancer predisposition pathological condition of low penetrance and possibly relative good prognosis. 


\section{References}

1. Sugiyama M and Atomi Y: Extrapancreatic neoplasms occur with unusual frequency in patients with intraductal papillary mucinous tumors of the pancreas. Am J Gastroenterol 94: 470-473, 1999.

2. Yamaguchi K, Ohuchida J, Ohtsuka T, Nakano K and Tanaka M: Intraductal papillary-mucinous tumor of the pancreas concomitant with ductal carcinoma of the pancreas. Pancreatology 2: 484-490, 2002.

3. Bassi C, Sarr MG, Lillemoe KD and Reber HA: Natural history of intraductal papillary mucinous neoplasms (IPMN): Current evidence and implications for management. J Gastrointest Surg 12: 645-650, 2008.

4. Choi MG, Kim SW, Han SS, Jang JY and Park YH: High incidence of extrapancreatic neoplasms in patients with intraductal papillary mucinous neoplasms. Arch Surg 141: 51-56, discussion 56, 2006.

5. Eguchi H, Ishikawa O, Ohigashi H, Tomimaru Y, Sasaki Y, Yamada T, Tsukuma H, Nakaizumi A and Imaoka S: Patients with pancreatic intraductal papillary mucinous neoplasms are at high risk of colorectal cancer development. Surgery 139: 749-754, 2006.

6. Baumgaertner I, Corcos O, Couvelard A, Sauvanet A, Rebours V, Vullierme MP, Hentic O, Hammel P, Lévy P and Ruszniewski P: Prevalence of extrapancreatic cancers in patients with histologically proven intraductal papillary mucinous neoplasms of the pancreas: A case-control study. Am J Gastroenterol 103: 2878-2882, 2008.

7. Yoon WJ, Ryu JK, Lee JK, Woo SM, Lee SH, Park JK, Kim YT and Yoon YB: Extrapancreatic malignancies in patients with intraductal papillary mucinous neoplasm of the pancreas: Prevalence, associated factors, and comparison with patients with other pancreatic cystic neoplasms. Ann Surg Oncol 15: 3193-3198, 2008.

8. Reid-Lombardo KM, Mathis KL, Wood CM, Harmsen WS and Sarr MG: Frequency of extrapancreatic neoplasms in intraductal papillary mucinous neoplasm of the pancreas: Implications for management. Ann Surg 251: 64-69, 2010.

9. Larghi A, Panic N, Capurso G, Leoncini E, Arzani D, Salvia R, Del Chiaro M, Frulloni L, Arcidiacono PG, Zerbi A, et al: Prevalence and risk factors of extrapancreatic malignancies in a large cohort of patients with intraductal papillary mucinous neoplasm (IPMN) of the pancreas. Ann Oncol 24: 1907-1911, 2013.

10. Kawakubo K, Tada M, Isayama H, Sasahira N, Nakai Y, Yamamoto K, Kogure H, Sasaki T, Hirano K, Ijichi H, et al: Incidence of extrapancreatic malignancies in patients with intraductal papillary mucinous neoplasms of the pancreas. Gut 60: 1249-1253, 2011.

11. Marchegiani G, Malleo G, D'Haese JG, Wenzel P, Keskin M, Pugliese L, Borin A, Benning V, Nilsson L, Oruc N, et al: Association between pancreatic intraductal papillary mucinous neoplasms and extrapancreatic malignancies. Clin Gastroenterol Hepatol 13: 1162-1169, 2015.
12. Tanaka M, Fernández-del Castillo C, Adsay V, Chari S, Falconi M, Jang JY, Kimura W, Levy P, Pitman MB, Schmidt CM, et al; International Association of Pancreatology: International consensus guidelines 2012 for the management of IPMN and MCN of the pancreas. Pancreatology 12: 183-197, 2012.

13. International Rules for Multiple Primary Cancers ICD-O. Third Edit. Internal Report, Lyon, IARC, 2004.

14. Matsuda T, Marugame T, Kamo K, Katanoda K, Ajiki W and Sobue T; Japan Cancer Surveillance Research Group: Cancer incidence and incidence rates in Japan in 2003: Based on data from 13 population-based cancer registries in the Monitoring of Cancer Incidence in Japan (MCIJ) Project. Jpn J Clin Oncol 39: 850-858, 2009

15. R Development Core Team: R: A language and environment for statistical computing. R Foundation for Statistical Computing, 2011.

16. Sullivan KM, Dean A and Soe MM: OpenEpi: A web-based epidemiologic and statistical calculator for public health. Public Health Rep 124: 471-474, 2009

17. World Medical A; World Medical Association: World Medical Association Declaration of Helsinki: Ethical principles for medical research involving human subjects. JAMA 310: 2191-2194, 2013

18. Tabuchi T, Ito Y, Ioka A, Miyashiro I and Tsukuma H: Incidence of metachronous second primary cancers in Osaka, Japan: Update of analyses using population-based cancer registry data. Cancer Sci 103: 1111-1120, 2012.

19. Chen T, Fallah M, Jansen L, Castro FA, Krilavicuite A, Katalinic A, Eisemann N, Emrich K, Holleczek B, Geiss K, et al; GEKID Cancer Survival Working Group: Distribution and risk of the second discordant primary cancers combined after a specific first primary cancer in German and Swedish cancer registries. Cancer Lett 369: 152-166, 2015.

20. Lee SY, Choi DW, Jang KT, Lee KT, Choi SH, Heo JS, Lee JK, Paik SW and Rhee JC: High expression of intestinal-type mucin (MUC2) in intraductal papillary mucinous neoplasms coexisting with extrapancreatic gastrointestinal cancers. Pancreas 32: 186-189, 2006

21. Hong SM, Omura N, Vincent A, Li A, Knight S, Yu J, Hruban RH and Goggins M: Genome-wide CpG island profiling of intraductal papillary mucinous neoplasms of the pancreas. Clin Cancer Res 18: 700-712, 2012.

22. Almoguera C, Shibata D, Forrester K, Martin J, Arnheim N and Perucho M: Most human carcinomas of the exocrine pancreas contain mutant c-K-ras genes. Cell 53: 549-554, 1988.

23. Schönleben F, Qiu W, Ciau NT, Ho DJ, Li X, Allendorf JD, Remotti HE and Su GH: PIK3CA mutations in intraductal papillary mucinous neoplasm/carcinoma of the pancreas. Clin Cancer Res 12: 3851-3855, 2006.

24. von Figura G, Fukuda A, Roy N, Liku ME, Morris Iv JP, Kim GE, Russ HA, Firpo MA, Mulvihill SJ, Dawson DW, et al: The chromatin regulator Brg1 suppresses formation of intraductal papillary mucinous neoplasm and pancreatic ductal adenocarcinoma. Nat Cell Biol 16: 255-267, 2014. 